\title{
Quantum time uncertainty in Schwarzschild-anti-de Sitter black holes
}

\author{
Pablo Galán, ${ }^{1, *}$ Luis J. Garay, ${ }^{2,1, \dagger}$ and Guillermo A. Mena Marugán ${ }^{1, \dagger}$ \\ ${ }^{1}$ Instituto de Estructura de la Materia, CSIC, Serrano 121, 28006 Madrid, Spain \\ ${ }^{2}$ Departamento de Física Teórica II, Universidad Complutense de Madrid, 28040 Madrid, Spain
}

(Received 12 March 2007; published 17 August 2007)

\begin{abstract}
The combined action of gravity and quantum mechanics gives rise to a minimum time uncertainty in the lowest order approximation of a perturbative scheme, in which quantum effects are regarded as corrections to the classical spacetime geometry. From the nonperturbative point of view, both gravity and quantum mechanics are treated on equal footing in a description that already contains all possible backreaction effects as those above in a nonlinear manner. In this paper, the existence or not of such minimum time uncertainty is analyzed in the context of Schwarzschild-anti-de Sitter black holes using the isolated horizon formalism. We show that from a perturbative point of view, a nonzero time uncertainty is generically present owing to the energy scale introduced by the cosmological constant, while in a quantization scheme that includes nonperturbatively the effects of that scale, an arbitrarily high time resolution can be reached.
\end{abstract}

DOI: 10.1103/PhysRevD.76.044014

PACS numbers: 04.60.-m, 03.65.Ta, 04.60.Kz, 04.70.Dy

\section{INTRODUCTION}

The emergence of a minimum time (or length) uncertainty in the presence of gravity is often analyzed by adopting perturbative approaches [1]. In standard quantum mechanics, the time evolution is subject to the fourth Heisenberg uncertainty relation: the time necessary to ensure that a system has evolved is larger than the inverse of the root mean square (rms) deviation of the energy distribution. Therefore, to improve the time sensitivity, one must consider states with an increasing energy uncertainty, reaching a perfect time resolution only if the energy is completely unknown. In general relativity, on the other hand, Einstein equations imply that an uncertainty in the energy of the system causes uncertainty in the geometry of spacetime and consequently in the measurement of time. In this way the uncertainty in the time evolution of a physical system is due to competing contributions from both quantum and gravitational origin: energy uncertainty must be large so that the quantum time uncertainty is small; energy uncertainty must be small so that spacetime geometry is not seriously disturbed. As a result, an infinite time resolution seems impossible in scenarios involving quantum and gravitational effects, and, indeed, several arguments indicate that time uncertainty cannot be made as small as desired in these kinds of perturbative approaches (at least in the next-to-leading-order approximation) [1-4]. However, it is far from clear whether this conclusion signals a fundamental phenomenon in quantum gravity or can be eluded by adopting a nonperturbative quantum description of the gravitational processes, a possibility that the models described in Refs. [5-7] actually suggest.

\footnotetext{
*galan@iem.cfmac.csic.es

luis.garay@fis.ucm.es

mena@iem.cfmac.csic.es
}

In those references it has been shown that some systems present in fact a nonvanishing time uncertainty when treated perturbatively, while the time resolution may become arbitrarily high if nonperturbative approaches are employed. It is remarkable that these fundamentally different behaviors show up in quantum systems that are sufficiently simple so as to allow full quantum perturbative and nonperturbative descriptions. Whether this is indeed a general feature of quantum gravitational systems or not still remains to be seen. Among these studies, we can find very different systems such as Einstein-Rosen waves [5] or the so-called doubly special relativity formalisms [6]. They share a common feature which is the existence of modified dispersion relations, in the sense that (nonlinear) redefinitions of the energy and also of momentum reveal themselves as natural tools to study these systems. Whether these redefinitions and modified dispersion relations are just tools or intrinsic characteristics of the system depends on the point of view-perturbative or not-that one is willing to adopt.

Black hole physics presents itself as a suitable scenario in which the possible appearance of a bound to time resolution can be tested. These ideas are further motivated by the existence of results that hint at the discreteness of the spectrum of geometric operators such as the area of the black hole horizon [8]. Although the discrete nature of these operators does not necessarily imply a minimum spacetime uncertainty, it certainly leads to a spacetime picture which is not continuous at small scales. A closely related issue which suggests the use of black holes to analyze the perturbative nature (or not) of minimum time uncertainties is that they saturate the amount of information that can be stored in a spacetime region of the same size [9], which, together with the ideas of the holographic principle, provides a nontrivial time-uncertainty lower bound [10]. 
In black hole physics, the definition of a notion of horizon energy generically rests on normalization conventions or global properties. As a consequence, the energy does not have a genuine unambiguous meaning, even when the horizon is associated with a global Killing field. For instance, in the simple case of event horizons in static and asymptotically flat spacetimes, the energy can be determined using conditions imposed in the asymptotic region, namely, that the global Killing field be normalized to the unity there. In this way one assigns the (numerical value of the) ADM energy to the horizon $[11,12]$. These types of criteria lose their relevance when horizons are analyzed quasilocally, so that the knowledge of the whole spacetime is not presumed $[12,13]$.

For the sake of simplicity, we will concentrate on the nonrotating case in absence of matter fields. Then, the energy of the horizon generates "on shell" time translations on the horizon along a suitable vector field which coincides with a null normal. This normal can be freely rescaled by a constant, which may even vary from one black hole solution to another. As we have commented, if the spacetime is static and asymptotically flat, one can eliminate this freedom by restricting oneself precisely to the null normal provided by the Killing field which has unit norm at infinity. This is not possible in general (for instance if there exists radiation in the exterior region or the metric fails to have an appropriate asymptotic symmetry). In order to analyze these sorts of systems, one is led to consider the notion of isolated horizons [12] which need not be Killing horizons nor require the entire spacetime history, as event horizons do. Isolated horizons are in equilibrium: no matter or radiation falls through them and their area remains constant. In this formalism, elaborated by Ashtekar et al., the energy of the isolated horizon can be defined using a Hamiltonian language. The problem of normalization of the time vector field which becomes the null normal on the horizon and defines its mass is still present. Indeed, there is an infinite family of parameter-dependent timelike vector fields each defining a consistent Hamiltonian evolution and a horizon energy. The possible generators of Hamiltonian transformations on the space of solutions (the covariant phase space) can be identified as acceptable elections of the horizon energy [13] or, with an alternate terminology, of the horizon mass function $\mu$.

The normalization of the time vector field is important because the surface gravity $\kappa$ depends on it. Given a horizon whose area is $A$, the mass function $\mu$ and the time vector field along which evolution takes place are closely related. Indeed, it can be shown that the evolution defined by some timelike vector field is Hamiltonian if and only if the first law of black hole dynamics holds, $\delta \mu=$ $(\kappa / 8 \pi) \delta A[13,14]$. But this still leaves a lot of freedom in the choice of normalization of the time vector and the mass function: any integrable function $\kappa(A)$ leads to an acceptable mass function $\mu(A)$ for the horizon.
In certain situations, the existence of different allowed mass functions can be thought of as the result of a modified normalization of the time vector that incorporates gravitational effects with respect to a background (e.g. a Schwarzschild spacetime). In particular, here we will focus our attention on the choice of mass function for black holes in a spacetime with a negative cosmological constant and no matter content - we will refer to them generically as Schwarzschild-anti-de Sitter (or Schwarzschild-AdS) black holes. We will study whether the presence of a scale fixed by the cosmological constant implies the emergence of a minimum time uncertainty when one (erroneously) assumes an effective quantum description corresponding to an asymptotically flat space as the starting point.

In a perturbative approach to the treatment of the cosmological constant, one would begin with a Schwarzschild black hole and the corresponding horizon mass defined through the conventional asymptotic normalization of the time vector field. Then, one would proceed to introduce the effect of the cosmological constant, deforming hence the spacetime geometry. This deformation would change the normalization of the time vector field and, subsequently, result in a change of the horizon mass function. If the modifications are incorporated perturbatively, one would generally obtain a series of successive corrections.

Alternatively, one could adopt a nonperturbative approach in which a nonlinear global redefinition of the mass and time parameters encompassing all the effects would be in order. With these premises, we will consider the different possibilities of describing the quantum evolution in terms of a parameter that corresponds either to the time naturally associated with a Schwarzschild background or to the time corresponding to the Schwarzschild-AdS black holes. The latter can be regarded as the natural time (on the horizon, or globally for static solutions as we will see) whose definition includes the effects of the cosmological constant. In this sense, we will refer to these two types of quantization as perturbative and nonperturbative, respectively, given the distinct philosophy in the use of background structures.

The rest of the paper is organized as follows. In the following section, we introduce the two different choices for the time and mass function in Schwarzschild-AdS black holes discussed above, and we describe the nonlinear relations between them. In Sec. III we discuss a quantum framework for the description of these black holes and analyze the time uncertainty when one adopts a perturbative scheme for the treatment of the effects of the cosmological constant, proving that this uncertainty cannot generally vanish. In addition, we study the behavior of the time uncertainty in sectors of physical states with small and large horizon mass compared with the scale provided by the cosmological constant. In Sec. IV we show that the time uncertainty can be made to vanish in a nonperturbative quantization, in contrast with the perturbative ap- 
proach. We present our conclusions and some further discussion in Sec. V. The Appendix is devoted to some technical issues concerning the calculation of Laplace transforms.

\section{MASS FUNCTIONS FOR SCHWARZSCHILD- ADS BLACK HOLES}

We consider spacetimes with no matter fields that present a single nonrotating isolated horizon as their "internal boundary" [13] and that, in principle, may be asymptotically flat or anti-de Sitter (AdS) at infinity, depending on whether we specialize to Schwarzschild or Schwarzschild-AdS black holes. In the latter case, we assume the existence of a negative cosmological constant. Then, given the surface gravity $\kappa$, which depends on the normalization of the time vector identified with the null normal of the horizon, the first law of black hole dynamics determines the horizon energy as a function of the horizon area $A$, as we mentioned in the introduction. On the covariant phase space, the horizon energy plays the role of the generator of time evolution on the horizon. The total Hamiltonian is composed of two terms: the horizon energy and the contribution at infinity, which generates asymptotic timelike translations [13]. Furthermore, if one restricts oneself to the sector of static solutions, the considered timelike vector field can be chosen equal to the global static Killing field. In this case, the total Hamiltonian must vanish for symmetry reasons, implying that the horizon energy coincides (in value) with the generator of asymptotic time translations [13]. But since there exists only one vacuum static solution for each value of the black hole area $A$, the mass function $\mu(A)$ for the black hole is totally determined provided that there is a preferred choice of normalization for the static Killing field at infinity. Note also that the mass function obtained in this way generates evolution on the horizon with respect to a time that, for the static solutions, coincides precisely with the normalized asymptotic time.

In the asymptotically flat case, it is natural to go to the rest frame of the Schwarzschild black hole and normalize the static Killing field to be the unit at infinity. The generator of time translations at infinity is then the ADM mass [11]. In the asymptotically AdS case, one can introduce a similar choice of asymptotic time by imposing a standard normalization on the canonical generators of the AdS group $[15,16]$ and arrive to a conserved energy that, in the (static) Schwarzschild-AdS spacetimes, provides the mass parameter of the black hole [17]. But if we have no access to the asymptotic regions and analyze the horizon quasilocally, we have no reason to choose one or the other of these normalizations without additional information apart from the condition that the first law be satisfied. We will call Schwarzschild and Schwarzschild-AdS the mass functions derived with these two different normalizations for obvious reasons. One of the aims of this paper is to explore the consequences of using a Schwarzschild mass function and its associated time parameter instead of their Schwarzschild-AdS counterparts even when a negative cosmological constant is present.

For completeness, let us recall that the static metric of Schwarzschild and Schwarzschild-AdS spacetimes can be, respectively, expressed in the well-known form

$$
\begin{aligned}
d s^{2}= & -\left[1-\frac{2 M}{r}\right] d T^{2}+\left[1-\frac{2 M}{r}\right]^{-1} d r^{2}+r^{2} d \Omega^{2}, \\
d s^{2}= & -\left[1-\frac{2 m}{r}+\frac{\Lambda}{3} r^{2}\right] d t^{2}+\left[1-\frac{2 m}{r}+\frac{\Lambda}{3} r^{2}\right]^{-1} d r^{2} \\
& +r^{2} d \Omega^{2}
\end{aligned}
$$

where $d \Omega^{2}$ is the metric on the unit two-sphere, $\Lambda>0$ is the absolute value of the negative cosmological constant, and $M$ and $m$ are the mass parameters of the respective black hole solutions. These mass parameters coincide in numerical value with the corresponding generators of asymptotic time translations (in $T$ and $t$, respectively) at infinity. For instance, in the asymptotically flat case, $M$ is the ADM mass of the Schwarzschild black hole. The horizon area is $A=4 \pi r_{h}^{2}$ in both cases, where $r_{h}$ is given by the positive zero of the diagonal time component of the metric.

For a Schwarzschild and a Schwarzschild-AdS black hole, respectively, the horizon mass $\mu(A)$ is hence

$$
M(A)=\sqrt{\frac{A}{16 \pi}}, \quad m(A)=\sqrt{\frac{A}{16 \pi}}+\frac{4}{3} \Lambda\left(\sqrt{\frac{A}{16 \pi}}\right)^{3} .
$$

The notation $M(A)$ and $m(A)$ emphasizes the fact that the numerical value of these functions coincide with the mass parameters $M$ and $m$ of the corresponding static solutions. In the following, we will not display explicitly this area dependence. The relation between the two considered mass functions is clearly

$$
m=M+\frac{4}{3} \Lambda M^{3} .
$$

It is worth remarking that $m$ and $M$ indeed coincide in the limit $\Lambda \rightarrow 0$. Abusing of the notation we will call $T$ and $t$ the times which parametrize the evolution generated by the mass functions $M$ and $m$ on the horizon, respectively. Remember that these times parametrize also the asymptotic time translations (with a convenient convention of signs) if one restricts oneself to static solutions, and that the normalization adopted at infinity would be the standard one for $T$ if the spacetime were asymptotically flat, while it is standard for $t$ if the asymptotic behavior is in fact AdS.

Let us then assume from now on that we are studying the horizon of a Schwarzschild-AdS black hole (quasilocally). We will refer to the times $t$ and $T$ as the physical and auxiliary ones, respectively, since they provide the AdS 
time and its counterpart for vanishing cosmological constant in the static sector, as we have commented. These times differ in a (solution-dependent, i.e. mass-dependent) constant factor which relates the two normalizations under consideration:

$$
t=V(M ; \Lambda) T .
$$

Obviously, we have chosen the same origin for both times, so that they vanish simultaneously. The surface gravities associated with these different normalizations of the time vector field are related by $\kappa_{t}=V^{-1} \kappa_{T}$. On the other hand, the first law imposes that the horizon mass in each case is determined by

$$
\delta m=\frac{\kappa_{t}}{8 \pi} \delta A, \quad \delta M=\frac{\kappa_{T}}{8 \pi} \delta A .
$$

Dividing both expressions, we obtain the factor $V$ :

$$
V(M ; \Lambda)=\left[\frac{\partial m}{\partial M}\right]^{-1}=\frac{1}{1+4 \Lambda M^{2}} .
$$

Note that this factor is strictly positive, so that the time relation (2.4) is a bijection from $\mathbb{R}$ to $\mathbb{R}$. Besides, $V$ becomes the unit when $\Lambda$ vanishes, so that the two analyzed times coincide in the limit of vanishing cosmological constant. Finally, notice that $V$ is not a polynomial in $\Lambda$, so that its Taylor expansion around $\Lambda=0$ would provide a (perturbative) series with an infinite number of terms.

\section{TIME UNCERTAINTY: PERTURBATIVE CASE}

We will now assume the existence of a quantum description for our covariant phase space corresponding to solutions in a vacuum with an internal nonrotating isolated horizon. We will not adhere to a particular quantization to try to keep our discussion as generic as possible, and rather base our analysis on general features of the quantum theory. In particular, we expect that the horizon area be represented by a quantum observable (with positive spectrum), since it is a well-defined (positive) quantity on the considered covariant phase space. This is known to be the case, for instance, in loop quantum gravity $[8,18]$. Therefore, also the Schwarzschild and SchwarzschildAdS mass functions, being functions of the horizon area, will be represented by quantum observables, which can be defined by means of the spectral theorem. Note also that, even if there exists a cosmological constant and the covariant phase space corresponds to asymptotically $\mathrm{AdS}$ spacetimes, the Schwarzschild mass function will still be well defined and what will cease to be applicable is just its physical interpretation in terms of the normalization of the time vector field at infinity for the static solutions.

In this section we will study the consequences of adopting a quantum description in which the parameter of the evolution at the horizon is the auxiliary time $T$ (i.e. the choice of time which would correspond to the standard asymptotic time for static solutions if the cosmological constant vanished). This would be the natural choice of time if one started the analysis obviating the presence of a cosmological constant and decided to incorporate it afterwards by perturbative means. If the reduction to the static sector of the covariant phase space is meaningful quantum mechanically, the time $T$ would play the role of the asymptotic time parameter in this reduced theory, but with a nonstandard (mass-dependent) normalization caused by a negligent account of the existence of a cosmological constant [19]. We leave to the next section the discussion of the case in which the evolution parameter is chosen to be the physical time $t$. The remarkable point is that if the role of evolution parameter is indeed assigned to the auxiliary time, the physical time is represented as a one-parameter family of quantum observables

$$
\hat{t}=\hat{V} T,
$$

where $\hat{V}$ can be constructed from the operator representing the Schwarzschild mass function as $\hat{V}:=V(\hat{M} ; \Lambda)$.

In order to calculate the uncertainty in the elapsed physical time $\hat{t}$ (setting the starting time for our observations at $t=T=0$ ), we employ the following procedure. Given a quantum state, one can measure the probability density of the operator $\hat{V}$. We call $\Delta V$ and $\langle\hat{V}\rangle$ its rms deviation and mean value, respectively. On the other hand (as in standard quantum mechanics), the value of the elapsed time $T$ can be deduced by analyzing the evolution of the probability densities of observables in the quantum state, with a resolution that cannot be better, for each given observable, than the lapse of time required for a change in its expectation value of the amount of the rms deviation. Therefore, this resolution is limited by the fourth Heisenberg relation. Via the analysis of collections of probability densities of observables, the above process will lead to a statistical distribution for the value of the parameter $T$ (regarded as a random variable), that will be described by a probability density $\rho(T)$. The corresponding mean value will be denoted by $\bar{T}$. According to our comments and recalling that the evolution in the parameter $T$ is generated by $\hat{M}$, the uncertainty $\Delta T$ of this distribution must satisfy the fourth Heisenberg relation $\Delta T \Delta M \geq 1 / 2$. A double average is hence involved in the calculation of $\Delta t$ since we have to calculate the quantum expectation value $\langle\cdot\rangle$ and also the statistical average over the value of $T$ :

$$
\begin{aligned}
(\Delta t)^{2} & =\int d T \rho(T)\left\langle T^{2} \hat{V}^{2}-\bar{T}^{2}\langle\hat{V}\rangle^{2}\right\rangle \\
& =(\bar{T} \Delta V)^{2}+(\Delta T \Delta V)^{2}+\langle\hat{V}\rangle^{2}(\Delta T)^{2} .
\end{aligned}
$$

Because the last expression is a sum of positive terms, the time uncertainty vanishes if and only if all of them are equal to zero. We show now that this will not happen at any generic time $\bar{T} \neq 0$ [20]. For this purpose, it is convenient to express Eq. (3.2) as 


$$
(\Delta t)^{2}=\bar{T}^{2}(\Delta V)^{2}+\left\langle\hat{V}^{2}\right\rangle(\Delta T)^{2} \geq \bar{T}^{2}(\Delta V)^{2}+\frac{1}{4} \frac{\left\langle\hat{V}^{2}\right\rangle}{(\Delta M)^{2}},
$$

where we have used the fourth Heisenberg relation.

In order for the uncertainty $\Delta t$ to vanish, the first term in the last inequality must be zero which, for $\bar{T} \neq 0$, implies that $\Delta V$ must vanish. But then the second term cannot vanish. To prove this, notice that the function $V$ given by Eq. (2.6) is strictly monotonic in $M \in \mathbb{R}^{+}$, so that it provides a one-to-one map. Thus, the spectral theorem assures that the eigenstates of the operators corresponding to $V$ and $M$ coincide, and the condition $\Delta V=0$ implies that $\Delta M=0$. On the other hand, since $V^{2}$ is a strictly positive function of $M, \hat{V}^{2}$ is a positive operator and hence $\left\langle\hat{V}^{2}\right\rangle$ does not vanish [21]. Thus, if $\Delta V$ becomes zero, the last term in inequality (3.3) gets unboundedly large. It is worth remarking that this result is completely general: $\Delta t$ does not vanish regardless of the choice of quantum state (provided that [21] is taken into account).

In the rest of this section we will analyze in detail the behavior of this nonvanishing time uncertainty in two different regimes which are, respectively, included in the sectors of quantum states with small and large (expectation values of the) Schwarzschild mass in comparison with the mass scale provided by the cosmological constant. We will also relate this behavior with suggested bounds for the time resolution that have appeared in the literature.

\section{A. Small mass and holographic uncertainty}

For small Schwarzschild masses with respect to the scale determined by the cosmological constant (namely for $4 \Lambda M^{2}<1$ ), expression (2.6) can be expanded as a Taylor series around the origin, obtaining

$$
V=\frac{1}{1+4 \Lambda M^{2}}=\sum_{n=0}^{\infty}\left(-4 \Lambda M^{2}\right)^{n}
$$

This series can also be viewed as a perturbative expansion of $V$ in terms of the cosmological constant for any given finite mass $M$. A direct calculation, taking the above series as the formal definition of $V$, gives then

$$
\begin{aligned}
\langle\hat{V}\rangle^{2} & =\sum_{n=0}^{\infty}(-4 \Lambda)^{n} \sum_{l=0}^{n}\left\langle\hat{M}^{2 n-2 l}\right\rangle\left\langle\hat{M}^{2 l}\right\rangle, \\
\left\langle\hat{V}^{2}\right\rangle & =\sum_{n=0}^{\infty}(n+1)(-4 \Lambda)^{n}\left\langle\hat{M}^{2 n}\right\rangle, \\
(\Delta V)^{2} & =\sum_{n=1}^{\infty}(-4 \Lambda)^{n}\left[n\left\langle\hat{M}^{2 n}\right\rangle-\sum_{l=1}^{n}\left\langle\hat{M}^{2 n-2 l}\right\rangle\left\langle\hat{M}^{2 l}\right\rangle\right] .
\end{aligned}
$$

Introducing these expressions in Eq. (3.3) we get

$$
\begin{aligned}
(\Delta t)^{2} \geq & \frac{1}{4(\Delta M)^{2}}+\sum_{n=1}^{\infty}(-4 \Lambda)^{n}\left[\frac{n+1}{4} \frac{\left\langle\hat{M}^{2 n}\right\rangle}{(\Delta M)^{2}}\right. \\
& \left.+\bar{T}^{2}\left(n\left\langle\hat{M}^{2 n}\right\rangle-\sum_{l=1}^{n}\left\langle\hat{M}^{2 n-2 l}\right\rangle\left\langle\hat{M}^{2 l}\right\rangle\right)\right] .
\end{aligned}
$$

Let us now restrict our discussion to the sector of quantum states with sufficiently small Schwarzschild mass, in the sense that

$$
1 \gg n \Lambda^{n}\left\langle\hat{M}^{2 n}\right\rangle, \quad \forall n \geq 1 .
$$

Alternatively, one can regard our discussion as corresponding to the limit of vanishing cosmological constant in the sector of quantum states with bounded even moments $\left\langle\hat{M}^{2 n}\right\rangle$ for the quantum observable $\hat{M}$ [22]. Conditions (3.7) are then satisfied when $\Lambda$ is sufficiently small.

In these circumstances, one can neglect the $\bar{T}$-independent corrections to the value of the (square) time uncertainty for zero cosmological constant, $1 /\left[4(\Delta M)^{2}\right]$. In principle, the $\bar{T}$-dependent corrections cannot be neglected if the auxiliary time can become unboundedly large, because $\bar{T}^{2}$ could compensate for the smallness of the factors containing powers of $\Lambda$ and the Schwarzschild mass. Nonetheless, we should expect from Eq. (3.5) that $\Delta V \approx 4 \Lambda \Delta\left(M^{2}\right)$ in the spirit of our small mass approximation. For instance, one can prove that this is actually so when inequality (3.7) holds if $\left\langle\hat{M}^{4}\right\rangle$ is of the same order or smaller than $\left[\Delta\left(M^{2}\right)\right]^{2}$ and besides

$$
\left[\Delta\left(M^{2}\right)\right]^{2} \gg n \Lambda^{n-2}\left\langle\hat{M}^{2 n}\right\rangle \quad \forall n>2 .
$$

We then reach the following (approximate) lower bound for the uncertainty $\Delta t$ :

$$
(\Delta t)^{2} \gtrsim \frac{1}{4(\Delta M)^{2}}+16 \Lambda^{2}\left[\Delta\left(M^{2}\right)\right]^{2} \bar{T}^{2} .
$$

Again, this equation can be interpreted as the Heisenberg bound for the time uncertainty when $\Lambda$ vanishes modified with the first significant perturbative correction in the limit of negligibly small cosmological constant [23].

We next define

$$
\omega:=\frac{\Delta\left(M^{2}\right)}{(\Delta M)^{2}} .
$$

Although $\omega$ and $\Delta M$ can be treated in principle as independent parameters in the Hilbert space of quantum states, inasmuch as they involve different moments of the quantum probability distribution for the observable $\hat{M}$ (the quantity $\Delta\left(M^{2}\right)$ involves $\left\langle\hat{M}^{4}\right\rangle$ whereas $\Delta M$ does not), one can argue that $\omega$, which is nonnegative by construction, has to be bounded from below by a strictly positive number. Indeed, on the one hand, since $M^{2}$ is a monotonic function of $M \in \mathbb{R}^{+}$, the spectral theorem ensures that $\Delta\left(M^{2}\right)$ vanishes if and only if so does $\Delta M$. In fact, it is possible to see that $(\Delta M)^{2}$ approaches zero faster than $\Delta\left(M^{2}\right)$. On the other hand, employing Eq. (3.7) one can 
show that, for each given value of $\Lambda, \Delta M$ is bounded from above in the considered sector of quantum states. Hence, $\omega$ cannot approach zero by letting $\Delta M$ diverge. As a result, one can convince oneself that there must exist a (possibly $\Lambda$-dependent) positive number $\omega_{0}>0$ such that $\omega \geq \omega_{0}$.

We then have

$$
(\Delta t)^{2} \gtrsim \frac{1}{4(\Delta M)^{2}}+16 \Lambda^{2} \omega_{0}^{2} \bar{T}^{2}(\Delta M)^{4} .
$$

This expression can be minimized with respect to its dependence in $\Delta M$, obtaining in this way the minimum time uncertainty at each instant of time $\bar{T}$ in the sector of states under study. The extremum at time $\bar{T}$ is reached on states with $(\Delta M)_{\min }^{-6}=128 \omega_{0}^{2} \Lambda^{2} \bar{T}^{2}$, and the corresponding minimum uncertainty is

$$
(\Delta t)_{\min }=\sqrt{3}\left(\frac{\Lambda \omega_{0} \bar{T}}{2}\right)^{1 / 3}
$$

which scales with the auxiliary time as $\bar{T}^{1 / 3}$.

It is worth remarking that this behavior of the time uncertainty is precisely the same that one finds by applying holographic arguments to black holes in quantum gravity [10]. Nevertheless, it is important to point out that our result depends on the type of quantum states that one chooses. We would find a generally different auxiliary time dependence for the time uncertainty if we considered states other than those satisfying the conditions commented above, which, in particular, imply that the even moments of the Schwarzschild mass are sufficiently small compared with the mass scale determined by the cosmological constant.

\section{B. Large mass and linear uncertainty}

We now analyze the time uncertainty on quantum states that present an approximate Gaussian distribution in the Schwarzschild mass, peaked around a large eigenvalue in Planck units. In principle, one might worry about the very assumption of the existence of such states, since we have preferred not to adhere to any specific quantization of our covariant phase space and therefore we cannot give precise statements about the spectrum of the Schwarzschild mass. For instance, from the discreteness of the area spectrum in loop quantum gravity [8], one would expect that a loop quantization of our system would lead to a discrete Schwarzschild mass. Nonetheless, for large values of the Schwarzschild mass function in Planck units (and then of the AdS mass, see Eq. (2.3)), one should expect the (semi)classical description of the black hole to be a fairly good approximation. Hence, the work hypothesis that the mass spectrum is almost continuous in the region of macroscopic black holes is a reasonable supposition, when not a requirement that should be imposed in order to select the physically admissible quantizations. On the other hand, we will also restrict the mass eigenvalue around which the quan- tum state peaks to be at least of the order of the mass scale provided by the cosmological constant.

In more detail, we are going to concentrate our discussion on quantum states whose associated probability distribution for the Schwarzschild mass has the approximate Gaussian form

$$
\bar{\rho}(M)=\frac{1}{N} e^{-[(M / \sigma)-\mu]^{2}}
$$

and allow $M$ to run over the positive real axis. The factor $N$ is a normalization constant, so that $\bar{\rho}$ is a normalized distribution. It is straightforward to see that

$$
N=\sigma \int_{-\mu}^{\infty} d u e^{-u^{2}}:=\sigma \Xi(\mu) .
$$

The function $\Xi(\mu)$ can be expressed in terms of the common error function [24]:

$$
\Xi(\mu)=\frac{\sqrt{\pi}}{2}[1+\operatorname{erf}(\mu)], \quad \operatorname{erf}(\mu)=\frac{2}{\sqrt{\pi}} \int_{0}^{\mu} d u e^{-u^{2}} .
$$

Remember that $\operatorname{erf}(\mu)$ tends to the unit if $\mu \rightarrow \infty$. On the other hand, $\mu$ is related with the mean value of the mass distribution measured in $\sigma$ units. Explicitly,

$$
\langle\hat{M}\rangle=\sigma\left[\mu+\frac{e^{-\mu^{2}}}{2 \Xi(\mu)}\right] .
$$

Hence, for large values of $\mu$, we have both that the mean value of $\hat{M}$ is large and that it coincides with $\sigma \mu$ up to negligible exponentially decreasing terms. Finally $\sigma$, apart from providing a unit of mass, would correspond to the rms deviation of a usual Gaussian were the variable $M$ defined on the entire real line, rather than on the positive real axis. Taking into account this restriction on the range of $M$, one can check that

$$
(\Delta M)^{2}=\frac{\sigma^{2}}{2}\left[1-\frac{\mu e^{-\mu^{2}}}{\Xi(\mu)}-\frac{e^{-2 \mu^{2}}}{2 \Xi(\mu)^{2}}\right] .
$$

Therefore, for large $\mu$ and up to exponentially small corrections, the rms deviation becomes $\sigma / \sqrt{2}$. In total, we see that the distribution (3.13) can be considered a well-peaked Gaussian around large values of the Schwarzschild mass (compared with the Planck mass) if $\mu \gg 1$ and $\sigma$ is not considerably large in Planck units.

With the probability distribution (3.13), one obtains for the operator $\hat{V}$

$$
\left\langle\hat{V}^{n}\right\rangle=\frac{1}{\sqrt{\lambda} \Xi(\mu)} \int_{-\gamma}^{\infty} d v \frac{e^{-v^{2} / \lambda}}{\left[1+(v+\gamma)^{2}\right]^{n}} .
$$

We have defined

$$
\lambda:=4 \Lambda \sigma^{2}, \quad \gamma:=\mu \sqrt{\lambda}, \quad v:=\sqrt{\lambda}\left[\frac{M}{\sigma}-\mu\right] .
$$


Note that expression (3.18) has the form of a Laplace integral. In the Appendix we show how to compute its asymptotic series expansions in powers of $\lambda$ in the limit of vanishing cosmological constant. In those computations, we treat $\gamma$ as a given number. Up to a constant numerical factor, this number is precisely the mean value of $\hat{M}$ expressed in terms of the mass scale provided by the cosmological constant (in the approximation of large $\mu$ ). Remember that we have assumed that these two masses are of the same order, so that we cannot neglect $\gamma$, even if we analyze the asymptotic limit $\lambda \rightarrow 0$.

So, we now specialize the calculations to the region of large black hole masses, where the consideration of the approximate Gaussian distributions (3.13) is justified, and study the situation of a very small cosmological constant with fixed $\gamma$. Under these circumstances, one can approximate the function $\Xi(\mu)$ to $\sqrt{\pi}$ and $\Delta M$ to $\sigma / \sqrt{2}$ by disregarding exponentially small terms. In addition, one gets the following leading-order contributions for the moments of $\hat{V}$ (see the Appendix for details):

$$
\left\langle\hat{V}^{2}\right\rangle \approx \frac{1}{\left(1+\gamma^{2}\right)^{2}}, \quad(\Delta \hat{V})^{2} \approx \lambda \frac{2 \gamma^{2}}{\left(1+\gamma^{2}\right)^{4}} .
$$

With all these results, one obtains from Eq. (3.3):

$$
(\Delta t)^{2} \geq \frac{1}{2\left(1+\gamma^{2}\right)^{2} \sigma^{2}}+\lambda \frac{2 \gamma^{2}}{\left(1+\gamma^{2}\right)^{4}} \bar{T}^{2} .
$$

The last term on the right-hand side has been conserved, even though it is of higher-order in $\lambda$ in comparison with the first one, because the auxiliary time can be unboundedly large. In this way, we obtain a time uncertainty that is always bounded from below by a positive constant contribution $1 /\left[\sqrt{2}\left(1+\gamma^{2}\right) \sigma\right]$ and that for large (auxiliary) times grows linearly with $\bar{T}$ (and thus with $\langle t\rangle$, see Eq. (2.4)).

\section{TIME UNCERTAINTY: NONPERTURBATIVE CASE}

We turn now to the discussion of the time uncertainty when one considers that the quantum evolution on the horizon is dictated by the Schwarzschild-AdS mass function and the corresponding evolution parameter is thus the physical time $t$. Remember that, if the reduction to the static sector of the covariant phase space is meaningful quantum mechanically, the physical time can be viewed in the reduced theory as the asymptotic AdS time parameter with the standard normalization at infinity.

The fundamental difference with respect to our analysis in the previous section is that now $t$ is not a one-parameter family of observables, but instead a genuine parameter. Its uncertainty is only limited now by the fourth Heisenberg relation, namely $\Delta t \Delta m \geq 1 / 2$.

As a consequence, in this nonperturbative quantum description, the resolution for the physical time is intrinsi- cally bounded if and only if the same happens for the AdS mass $m$. The conclusion does not depend on other details of the system. The only relevant point is whether the range of the horizon energy is bounded or not. This range is determined in the quantum theory by the spectrum of the observable $\hat{m}:=m(\hat{A})$ obtained from Eq. (2.2). Since the classical function $m(A)$ tends to infinity when so does $A$, the spectrum of the AdS mass will be indeed unbounded if the same happens with the black hole area spectrum. This last assumption is certainly reasonable, since one should expect no important quantum deviations from the (semi)classical description for macroscopic black holes with large horizon areas (for instance, the area spectrum is known to be unbounded in loop quantum gravity [8]). Therefore, a finite time resolution is not a necessary consequence of the quantization of the system. At least in this nonperturbative framework, the quantum resolution in the physical time can be made as large as desired.

\section{CONCLUSION}

We have argued that, for Schwarzschild-AdS black holes, a perturbative approach to quantization gives rise to a (time and quantum-state dependent) lower bound on the time uncertainty, while nonperturbative treatments allow for arbitrarily high time resolutions. This conclusion has been reached by analyzing the quantization of a Schwarzschild-AdS black hole using different mass functions for the horizon, each of them generating evolution on the horizon with respect to a different time parameter.

We started with the mass function that, for static solutions, would correspond to Schwarzschild black holes with a standard normalization at infinity of the time vector field as if, instead of dealing with a Schwarzschild-AdS black hole, we had an asymptotically flat spacetime. The presence of the cosmological constant was then introduced as a contribution to the spacetime energy which modifies the normalization of the time vector field and hence the mass function, leading to effects that can be described by a perturbative power series in the cosmological constant (see e.g. Eq. (3.4)).

On the other hand a nonperturbative quantization has also been carried out. In this approach, the mass function for the horizon is that obtained with the standard normalization of the time vector field at infinity for the kind of spacetimes that we are truly studying, i.e. SchwarzschildAdS black holes. By adopting this choice of the mass function, one is naturally including the effects of the cosmological constant in our description from the beginning. The quantizations based on the two commented choices of the mass function are not equivalent, because the physical time, which reproduces the AdS time on static solutions, is described in one case as a parameter, while in the other case is represented as a one-parameter family of observables. Indeed we have seen that the two quantization schemes yield different physical results. 
In the nonperturbative approach, time uncertainty is just dictated by the fourth Heisenberg uncertainty principle and therefore presents no lower bound, because the AdS mass is an unbounded function of the horizon area, and (it seems reasonable to assume that) it is represented by an unbounded operator in the quantum theory.

From the perturbative point of view, on the other hand, we have seen that the physical time uncertainty can never be made equal to zero. In the limit of a negligibly small cosmological constant, this uncertainty can be expanded in an asymptotic power series. We have shown that the minimum time uncertainty, although universally nonvanishing, has a behavior that strongly depends on the quantum state. Indeed we have considered different quantum states which describe black holes with small and large mass with respect to the mass scale supplied by the cosmological constant. These lead to different types of time dependence, which have the general form $\Delta t_{\min } \sim\langle t\rangle^{1-\delta}$ (in Planck units) with $0 \leq \delta \leq 1$, as discussed for instance in Ref. [10]. The specific classes of quantum states that we have analyzed illustrate the behaviors for $\delta=1,2 / 3$, and 0 . These kinds of uncertainties correspond, respectively, to the cases of a constant (Planck) minimum time uncertainty, a holographic-principle type of uncertainty, and a constant relative time uncertainty (so that the absolute one is cumulative and grows linearly with time).

On the other hand, one might try to extend our analysis to the case of Schwarzschild-de Sitter black holes. A standard normalization of the time vector [16] would lead to a correspondence similar to Eq. (2.3), but with a flip of sign in the last term, arising now from a positive cosmological constant. As a consequence, the relation between the two considered masses would be one-to-one only in a bounded interval of positive masses including the origin. This restriction on the allowed masses, that would affect the analysis, is not casual: the upper bound in the Schwarzschild-de Sitter mass is just the value for which one gets a static solution with coincident black hole and cosmological horizons. Furthermore, the presence of a cosmological horizon casts shadows on the naturalness of the standard normalization adopted for the time vector [16] and, in any case, makes unavailable a physical interpretation in terms of asymptotic timelike translations on static solutions. Owing to these reasons, we have restricted our discussion to Schwarzschild-AdS black holes.

Finally, let us emphasize that the fact that a nonvanishing minimum uncertainty only appears perturbatively, not just in our analysis of Schwarzschild-AdS black holes, but also in other systems which allow a full quantization both from the perturbative and nonperturbative points of view, casts doubts on the belief that universal time resolution bounds, applicable to generic quantum states, represent an essential feature of a full quantum theory of gravity and suggests the possibility that they are indeed eluded when a nonperturbative quantization of these systems is carried out.

\section{ACKNOWLEDGMENTS}

The authors want to thank V. Aldaya and C. Barceló for fruitful conversations and enlightening discussions. P. G. is also very thankful to F. Barbero and J. M. Martín-García for their valuable help. P. G. gratefully acknowledges the financial support provided by the I3P framework of CSIC and the European Social Fund. This work was supported by funds provided by the Spanish MEC Projects No. FIS200505736-C03-02 and No. FIS2006-26387-E.

\section{APPENDIX: CALCULATION OF LAPLACE INTEGRALS}

In this appendix we explain the computation of the mean value of the operators $\hat{V}$ and $\hat{V}^{2}$ introduced in Sec. III, restricting the quantum states to have the Gaussian behavior given in Eq. (3.13). We will obtain asymptotic expansions for these quantities in powers of the cosmological constant, assuming that this constant is small.

Expression (3.18) for the mean values of powers of $\hat{V}$ is a Laplace integral. To compute it, we split each integral into two parts. One is integrated from $-\gamma$ to 0 and the other from zero to infinity. For the first part, we make the change of variable $v=-\sqrt{z}$, whereas for the second part we make $v=\sqrt{z}$. Thus, we obtain

$$
\begin{aligned}
\left\langle\hat{V}^{n}\right\rangle & =\frac{1}{\Xi(\mu)} \frac{1}{\sqrt{\lambda}}\left[I_{n-}(z)+I_{n+}(z)\right], \\
I_{n \mp}(z) & =\int_{0}^{r_{\mp}} \frac{d z e^{-z / \lambda}}{2 \sqrt{z}\left[1+(\gamma \mp \sqrt{z})^{2}\right]^{n}},
\end{aligned}
$$

with $r_{-}=\gamma^{2}$ and $r_{+}=\infty$.

These integrals belong to a large family of the form

$$
I(z)=\int_{0}^{r} d z F(z) e^{-\Omega z}, \quad r>0 .
$$

In the limit $\Omega \rightarrow+\infty$, Watson's lemma [25] gives the whole asymptotic expansion of any integral of this kind provided that $F(z)$ is continuous in the interval $0 \leq z \leq r$ and admits an asymptotic series expansion of the type

$$
F(z)=z^{\alpha} \sum_{k=0}^{\infty} a_{k} z^{\beta k}, \quad z \rightarrow 0^{+}
$$

It is necessary that $\alpha>-1$ and $\beta>0$ for the integral to converge at $z=0$. In addition, if $r=+\infty$, one must have $F(z) \ll e^{q z}(z \rightarrow+\infty)$ for some positive constant $q$. If the previous conditions are fulfilled,

$$
I(z) \approx \sum_{k=0}^{\infty} \frac{a_{k} \Gamma(\alpha+\beta k+1)}{\Omega^{\alpha+\beta k+1}}, \quad \Omega \rightarrow+\infty .
$$

Here, the symbol $\Gamma$ represents the Gamma function [24]. 
In our case, we have in the integrands

$$
F_{n \mp}(z)=\frac{1}{2 \sqrt{z}} \frac{1}{\left(1+\gamma^{2}\right)^{n}}\left(1 \mp \frac{2 \gamma \sqrt{z}}{1+\gamma^{2}}+\frac{z}{1+\gamma^{2}}\right)^{-n} .
$$

If we now call

$$
x=\frac{\gamma}{\sqrt{1+\gamma^{2}}}, \quad y=\frac{\sqrt{z}}{\sqrt{1+\gamma^{2}}},
$$

we realize that the factor in the parenthesis of the previous expression is the generating function of the Chebyshev polynomials of the second kind, $U_{k}(x)$ [24]:

$$
G(x, \mp y)=\frac{1}{1-2 x( \pm y)+y^{2}}=\sum_{k=0}^{\infty} U_{k}(x)( \pm y)^{k} .
$$

Therefore, it is straightforward to find the asymptotic series expansion of the functions $F_{n \mp}$. According to Watson's lemma, the upper limit of integration $r$ does not affect the asymptotic power series expansion of the integral. So, we can first sum the functions $F_{n-}$ and $F_{n+}$ to obtain a unique asymptotic series for the integrand and then apply formula (A4).

For $n=1$, for instance, we split the series expansion of $G(x, \mp y)$ in even and odd powers of $y$. Obviously, the contributions of all odd powers cancel out when we sum $G(x, y)$ and $G(x,-y)$. We hence get

$$
F_{1-}(z)+F_{1+}(z)=\frac{1}{\sqrt{z}} \sum_{k=0}^{\infty} \frac{U_{2 k}(x)}{\left(1+\gamma^{2}\right)^{k+1}} z^{k} .
$$

By comparing this with Eq. (A3) we find

$$
\alpha=-\frac{1}{2}, \quad \beta=1, \quad a_{k}=\frac{U_{2 k}(x)}{\left(1+\gamma^{2}\right)^{k+1}} .
$$

Introducing these values in Eq. (A4) with $\Omega=\lambda^{-1}$ :

$$
I_{1-}(z)+I_{1+}(z)=\sqrt{\lambda} \sum_{k=0}^{\infty} \frac{\Gamma(k+1 / 2)}{\left(1+\gamma^{2}\right)^{k+1}} \lambda^{k} U_{2 k}(x),
$$

where

$$
\Gamma(k+1 / 2)=\frac{(2 k-1) ! !}{2^{k}} \sqrt{\pi},
$$

and we adopt the convention $(-1) ! !=1$. Besides, in the sector of infinite black hole mass $(\mu \rightarrow \infty)$, we can write $\Xi(\mu) \approx \sqrt{\pi}$ up to negligible exponential corrections. Thus

$$
\langle\hat{V}\rangle \approx \frac{1}{1+\gamma^{2}} \sum_{k=0}^{\infty}\left[\frac{\lambda}{2\left(1+\gamma^{2}\right)}\right]^{k}(2 k-1) ! ! U_{2 k}(x) .
$$

For $n=2$, repeating the above steps, Watson's lemma leads to

$$
\begin{aligned}
\left\langle\hat{V}^{2}\right\rangle \approx & \frac{1}{\left(1+\gamma^{2}\right)^{2}} \sum_{p=0}^{\infty}\left[\frac{\lambda}{2\left(1+\gamma^{2}\right)}\right]^{p} \\
& \times(2 p-1) ! ! \sum_{k=0}^{2 p} U_{k}(x) U_{2 p-k}(x) .
\end{aligned}
$$

Finally, let us recall that the first Chebyshev polynomials of the second kind are $U_{0}(x)=1$ and $U_{1}(x)=2 x$.
[1] L. J. Garay, Int. J. Mod. Phys. A 10, 145 (1995); and references therein.

[2] T. Padmanabhan, Classical Quantum Gravity 4, L107 (1987).

[3] G. Amelino-Camelia, Mod. Phys. Lett. A 9, 3415 (1994); 11, 1411 (1996); 13, 1319 (1998).

[4] G. Amelino-Camelia, Phys. Rev. D 62, 024015 (2000); Y. J. Ng and H. van Dam, Found. Phys. 30, 795 (2000).

[5] J.F. Barbero G., G.A. Mena Marugán, and E. J.S. Villaseñor, Phys. Rev. D 69, 044017 (2004).

[6] P. Galán and G. A. Mena Marugán, Phys. Rev. D 70, 124003 (2004).

[7] P. Galán and G. A. Mena Marugán, Phys. Rev. D 72, 044019 (2005).

[8] C. Rovelli and L. Smolin, Nucl. Phys. B442, 593 (1995); B456, 753 (1995); A. Ashtekar and J. Lewandowski, Classical Quantum Gravity 14, A55 (1997).

[9] S. Lloyd, Nature (London) 406, 1047 (2000).

[10] Y. J. Ng, Mod. Phys. Lett. A 18, 1073 (2003); arXiv:gr-qc/ 0401015; Y. J. Ng and H. van Dam, Int. J. Mod. Phys. A 20, 1328 (2005).
[11] R. M. Wald, General Relativity (Chicago Press, Chicago, 1984).

[12] A. Ashtekar, A. Corichi, and K. Krasnov, Adv. Theor. Math. Phys. 3, 419 (2000); A. Ashtekar et al., Phys. Rev. Lett. 85, 3564 (2000); A. Ashtekar, C. Beetle, and S. Fairhurst, Classical Quantum Gravity 17, 253 (2000); A. Ashtekar, C. Beetle, and J. Lewandowski, Phys. Rev. D 64, 044016 (2001).

[13] A. Ashtekar, S. Fairhurst, and B. Krishnan, Phys. Rev. D 62, 104025 (2000).

[14] We adopt units in which $\hbar=c=G=1$, with $\hbar$ being Planck constant, $G$ Newton constant, and $c$ the speed of light.

[15] M. Henneaux and C. Teitelboim, Commun. Math. Phys. 98, 391 (1985); J. D. Brown and M. Henneaux, Commun. Math. Phys. 104, 207 (1986).

[16] A. Corichi and A. Gomberoff, Phys. Rev. D 69, 064016 (2004).

[17] A. Ashtekar and S. Das, Classical Quantum Gravity 17, L17 (2000).

[18] A. Ashtekar, J.C. Baez, and K. Krasnov, Adv. Theor. 
Math. Phys. 4, 1 (2000).

[19] Some references where the quantization of the static Schwarzschild solutions is studied are J. Makela and P. Repo, Phys. Rev. D 57, 4899 (1998); J. Makela, P. Repo, M. Luomajoki, and J. Piilonen, Phys. Rev. D 64, 024018 (2001); C. Vaz and L. Witten, Phys. Rev. D 60, 024009 (1999).

[20] This implies a nonvanishing uncertainty in any physical time interval, since we have identified the zero time with the instant at which we start the measurement process.

[21] We consider states with $\langle\hat{M}\rangle<\infty$ and, if needed, take on them the limit $\Delta M \rightarrow 0$.
[22] It suffices that there exists a positive constant $C$ such that $\left\langle\hat{M}^{2 n}\right\rangle<C^{n} \quad \forall n \geq 1$.

[23] For this, we have assumed that the time $\bar{T}$ can be large and restricted ourselves to states with bounded expectation values $\left\langle\hat{M}^{2 n}\right\rangle$ as explained in [22].

[24] I. S. Gradshteyn and I. M. Ryzhik, in Table of Integrals, Series and Products, edited by A. Jeffrey and D. Zwillinger (Academic Press, San Diego, 2000), 6th ed.

[25] C. M. Bender and S. A. Orszag, Advanced Mathematical Methods for Scientists and Engineers (Springer-Verlag, New York, 1999). 